KUCP-0110

OHU-9710

UT-790

quant-ph/9710064

\title{
Valleys in Quantum Mechanics
}

\author{
Hideaki AoYAma $^{\dagger}$, Hisashi KıKUCHI ${ }^{\dagger \dagger}$, Ikuo OKOUCHI ${ }^{\ddagger}$, \\ Masatoshi $\mathrm{SATO}^{\star}$ and Shinya WADA ${ }^{\ddagger}$ \\ ${ }^{\dagger}$ Department of Fundamental Sciences, \\ Faculty of Integrated Human Studies, \\ Kyoto University, Kyoto 606-01, Japan \\ aoyama@phys.h.kyoto-u.ac.jp \\ ${ }^{\dagger}$ Ohu University, Koriyama 963, Japan \\ kikuchi@yukawa.kyoto-u.ac.jp \\ $\ddagger$ Graduate School of Human and Environmental Studies, \\ Kyoto University, Kyoto 606-01, Japan \\ dai@phys.h.kyoto-u.ac.jp, shinya@phys.h.kyoto-u.ac.jp \\ ${ }^{\star}$ Department of Physics, University of Tokyo, Tokyo 113, Japan \\ msato@hep-th.phys.s.u-tokyo.ac.jp
}

October, 1997

\begin{abstract}
Conventionally, perturbative and non-perturbative calculations are performed independently. In this paper, valleys in the configuration space in quantum mechanics are investigated as a way to treat them in a unified manner. All the known results of the interplay of them are reproduced naturally. The prescription for separating the non-perturbative contribution from the perturbative is given in terms of the analytic continuation of the valley parameter. Our method is illustrated on a new series of examples with the asymmetric doublewell potential. We obtain the non-perturbative part explicitly, which leads to the prediction of the large order behavior of the perturbative series. We calculate the first 200 perturbative coefficients for a wide range of parameters and confirm the agreement with the prediction of the valley method.
\end{abstract}


In this paper, we report on "valleys" in the configuration space of the pathintegral. The valley is a series of configurations which connects a classical minimum (vacuum) with an instanton-like configuration. As will be shown in the following, it reveals how non-perturbative effects interfere with perturbative effects.

The perturbative expansion is the most common method to analyze models in quantum mechanics or quantum field theories. Its predictive power is, however, in doubt in a strict sense, for the perturbative series is known to be divergent in most of the quantum systems [1]. Models in quantum mechanics have been intensively studied in order to understand the problem and to obtain physical predictions [2]. The symmetric double-well potential provides a very useful testing ground in this respect [3]. Although the energy spectrum must be real by definition, the naive application of the Borel-sum method to the perturbation series results in a complex spectrum. The interplay of non-perturbative effect, the tunneling between the wells, was inferred to resolve this complexity [4, 5].

Our analysis sheds a new light on this topic: First, it explains naturally why the Borel singularity of the perturbation theory is canceled by the nonperturbative contribution induced by instantons, or more generally, valley-instantons. The interplay of the perturbative and non-perturbative contribution can be understood by the decomposition of the integral contour for the collective coordinate of the valley. Second, it predicts the large order behaviors of the perturbative series for the asymmetric double-well model, which interestingly defeats widely held beliefs that the perturbative series would be Borel-summable for states without quantum tunneling [6]. The valley-instanton [7, 8] provides us with the basis for the path-integral analysis. Some assumptions that underlie our analysis are tested by comparing our theoretical predictions with the numerical and exact calculations of the perturbative series.

The model we study is a one-dimensional system with coordinate $q$, with the following Hamiltonian;

$$
\begin{aligned}
H & =-\frac{1}{2} \frac{d^{2}}{d q^{2}}+V(q), \\
V(q) & =\frac{1}{2} q^{2}(1-g q)^{2}-\epsilon g q,
\end{aligned}
$$

where $g(>0)$ is a coupling constant and $\epsilon(>0)$ is a parameter which represents the asymmetry of the potential. For $\epsilon g^{2}<\sqrt{3} / 18$, this potential has two minima. Although our consideration is not restricted to the special kind of model, the reason we adopt the model is that it becomes supersymmetric at the special values of $\epsilon$. For $\epsilon=1,2$, this model is known to be supersymmetric [9, 10, 11]. The non-renormalization theorem makes clear the relation between the perturbative contribution and the non-perturbative one and gives a rich structure. We will see that the result of the valley analysis agrees with the predictions of the supersymmetry. 
In the case of $\epsilon=0$, the simplest example of the valley represents an instantonanti-instanton pair. It approaches to the infinitely separated instanton-antiinstanton configuration at one end and has a vacuum configuration at the other end; namely, as the instanton and the anti-instanton approach each other, the action decreases smoothly, and finally when they coincide, they annihilate each other and the configuration becomes the vacuum. A precise definition of the valley is given by the valley equation [12, 13]. The valley equation which we adopt here is

$$
\int d \tau^{\prime} \frac{\delta^{2} S}{\delta q(\tau) \delta q\left(\tau^{\prime}\right)} \cdot \frac{\delta S}{\delta q\left(\tau^{\prime}\right)}=\lambda \frac{\delta S}{\delta q(\tau)}
$$

where $S$ is the action and $\lambda$ is a constant [13]. The configuration which satisfies this equation extremizes the norm of the gradient vector $\int d \tau(\delta S / \delta q)^{2}$ on the contour plane with $S$ fixed. Then, if we identify the value of the action as the "height" of the configuration, this configuration is in a "valley" in the configuration space. The instanton-anti-instanton pair is not a classical solution, but it can be defined as a solution of the valley equation [13]. When instanton and anti-instanton are well-separated from each other, the collective coordinates of the valley are the relative distance between them and the center of mass.

The power of the valley equation is demonstrated in the case of $\epsilon \neq 0$. For this case, there is no instanton-like classical solution which starts from one minimum of the potential and ends at the other minimum. But such a solution exists for the valley equation (2) with $\lambda$ set to zero [7]. We dub the solution which starts from the (right) left minimum as the (anti-)valley-instanton. When $\epsilon g^{2}$ is small, the valley-instanton for the present model may be constructed in a similar way as given in [7];

$$
q(\tau)=\left\{\begin{array}{lc}
\epsilon g+\frac{1}{g} e^{\omega_{+} \tau}+\frac{3 \epsilon g}{\omega_{+}} \tau e^{\omega_{+} \tau}+\cdots & \text { if } \tau \ll-1 ; \\
\frac{1}{g} \frac{1}{1+e^{-\tau}}+\epsilon g+\cdots & \text { if }-1 / \epsilon g^{2} \ll \tau \ll 1 / \epsilon g^{2} ; \\
\frac{1}{g}+\epsilon g-\frac{1}{g} e^{-\omega_{-} \tau}-\frac{3 \epsilon g}{\omega_{-}} \tau e^{-\omega_{-} \tau}+\cdots & \text { if } \tau \gg 1,
\end{array}\right.
$$

where $\omega_{ \pm}=1 \mp 3 \epsilon g^{2}+\cdots$. When $\epsilon$ goes to zero, the (anti-)valley-instanton smoothly converges to the ordinary (anti-)instanton. This gives a natural extension of the instanton-anti-instanton pair in the case of $\epsilon \neq 0$. There are two types of the valleys, one made of a valley-instanton-anti-valley-instanton pair and the other of an anti-valley-instanton-valley-instanton pair [7]. We call the former as I-A valley and the latter A-I valley.]

\footnotetext{
${ }^{1}$ An interesting property of the I-A valley (A-I valley) is that it contains the bounce solution if $\epsilon>0(\epsilon<0)$. In most theories, the bounce solution is a signal for instability, then the
} 
Now let us evaluate the transition amplitude from the left minimum $\left|0_{L}\right\rangle$ to itself

$$
Z=\lim _{T \rightarrow \infty}\left\langle 0_{L}\left|e^{-H T}\right| 0_{L}\right\rangle
$$

in the background of the I-A valley. For simplicity, we focus mainly on the case of $\epsilon=0$. Except for the collective coordinate of the valley, the path integral can be performed by the Gaussian integral. Then we obtain

$$
Z=\lim _{T \rightarrow \infty} \text { const. } \times \frac{T}{\sqrt{g^{2}}} \int_{0}^{T} d R e^{-S(R) / g^{2}},
$$

where $R$ is the collective coordinate of the valley corresponding to the relative distance between the valley-instanton and the anti-valley-instanton, and $S(R) / g^{2}$ is the action of the valley. A factor $T / \sqrt{g^{2}}$ results from the integration of the collective coordinate for the translational symmetry. When $\epsilon=0$, the action of the valley behaves as 13

$$
S(R)= \begin{cases}\frac{R^{2}}{2} & \text { for } R \rightarrow 0 \\ \frac{1}{3}-2 e^{-R} & \text { for } R \rightarrow \infty\end{cases}
$$

The valley at $R=0$ is the vacuum and at $R \sim \infty$ the well-separated instantonanti-instanton pair; the term $1 / 3$ in Eq.(6) is their action and $-2 e^{-R}$ is the interaction between them. If we change the integration variable $R$ to $t=S(R)$, the amplitude (5) becomes

$$
Z=\lim _{x \rightarrow 1 / 3} \text { const. } \times \frac{T}{\sqrt{g^{2}}} \int_{C_{V}} d t F(t) e^{-t / g^{2}},
$$

where $x=S(T), C_{V}=[0, x]$, and $F(t)$ is the Jacobian. From Eq.([6), it is found that the Jacobian behaves as

$$
F(t)= \begin{cases}\frac{1}{\sqrt{2 t}} & \text { for } t \rightarrow 0 \\ \frac{1}{1 / 3-t} & \text { for } t \rightarrow \frac{1}{3}\end{cases}
$$

existence of the bounce solution in our stable system was somewhat mysterious. In our valley, the integration of the negative mode of the bounce turns out to be the integration of the collective coordinate of the valley which corresponds to the relative distance between valleyinstanton and anti-valley-instanton and cause no instability in the energy spectrum [7].

${ }^{2}$ When the instanton and anti-instanton are not far enough apart from each other, there is no natural definition of $R$. We define $R$ so as to give Eq.(5) and (6) . 
To obtain the full form of the Jacobian, the detailed analysis of the valley is needed. Instead, we simply assume that the full form of the Jacobian is given by

$$
F(t)=\frac{f(t)}{\sqrt{2 t}(1 / 3-t)},
$$

where $f(t)$ is an analytic function that satisfies

$$
f(0)=\frac{1}{3}, \quad f(1 / 3)=\sqrt{\frac{2}{3}} .
$$

No more details of $f(t)$ are needed in the following analysis.

The integral (17) contains both the perturbative contribution at $t \sim 0$ and the non-perturbative one at $t \sim 1 / 3$. To separate the perturbative and nonperturbative contributions, we deform the contour $C_{\mathrm{V}}$ to the sum of $C_{\mathrm{P}}$ and $C_{\mathrm{NP}}$ as is shown in Fig.1. Then the amplitude becomes

$$
\begin{aligned}
Z= & \lim _{x \rightarrow 1 / 3} \text { const. } \times \frac{T}{\sqrt{g^{2}}} \int_{C_{\mathrm{P}}} d t F(t) e^{-t / g^{2}} \\
& +\lim _{x \rightarrow 1 / 3} \text { const. } \times \frac{T}{\sqrt{g^{2}}} \int_{C_{\mathrm{NP}}} d t F(t) e^{-t / g^{2}} .
\end{aligned}
$$

Note that there is a significant resemblance between the first term on the righthand side and the formal Borel-summation of the perturbation series. The singularity of $F(t)$ at $t=1 / 3$ is the same as that of the Borel function, which indicates the non-Borel-summability of the perturbation series. One of the present authors conjectured that this decomposition is essential to understand the interplay of the instanton effect to the perturbative calculation [14]. We identify that the first term of Eq.(11) is the formal Borel-summation of the perturbative series and second term is the non-perturbative one. We show that this decomposition naturally reproduce all the known results of the interplay of them. We also present the predictions of the conjecture and test them. We denote the first term of Eq.(11) as $Z_{\mathrm{P}}$ and the second term as $Z_{\mathrm{NP}}$.

An immediate consequence of the decomposition is

$$
\operatorname{Im} Z_{\mathrm{P}}+\operatorname{Im} Z_{\mathrm{NP}}=0 \text {. }
$$

This is because $Z=Z_{\mathrm{P}}+Z_{\mathrm{NP}}$ is real. This simple equation explains why the imaginary part of the formal Borel-summation of the perturbation series is canceled by that of the instanton contribution. At the same time, it also shows that non-zero imaginary part of the non-perturbative contribution is a necessary and sufficient condition for the non-Borel-summability of the perturbative expansion. Furthermore, when the imaginary part is not zero, we can predict the large order behavior of the perturbative contribution from the dispersion relation. To this 
end, we examine the analyticity of $Z_{\mathrm{P}}\left(g^{2}\right)$ in the complex $g^{2}$-plane. When the phase of $g^{2}$ changes to $2 \pi$, the perturbative amplitude $Z_{\mathrm{P}}\left(g^{2}\right)$ changes as

$$
Z_{\mathrm{P}}\left(g^{2}\right) \rightarrow Z_{\mathrm{P}}\left(g^{2} e^{2 \pi i}\right)=Z_{\mathrm{P}}\left(g^{2}\right)-2 i \operatorname{Im} Z_{\mathrm{P}}\left(g^{2}\right)
$$

then, $Z_{\mathrm{P}}\left(g^{2}\right)$ has a cut on the real axis in the complex $g^{2}$ plane. This is the only singularity near the origin. Thus the dispersion relation becomes

$$
\begin{aligned}
Z_{\mathrm{P}}\left(g^{2}\right) & =\frac{1}{\pi} \int_{0}^{\infty} d z \frac{\operatorname{Im} Z_{\mathrm{P}}(z)}{z-g^{2}}+\cdots \\
& =-\frac{1}{\pi} \int_{0}^{\infty} d z \frac{\operatorname{Im} Z_{\mathrm{NP}}(z)}{z-g^{2}}+\cdots,
\end{aligned}
$$

where we have neglected the contribution from the singularity far from the origin. If we formally expand the integrand, we obtain the following: ${ }^{3}$

$$
Z_{\mathrm{P}}\left(g^{2}\right)=-\frac{1}{\pi} \sum_{m=0}^{\infty} \int_{0}^{\infty} d z \frac{\operatorname{Im} Z_{\mathrm{NP}}(z)}{z^{m+1}} g^{2 m}+\cdots
$$

When $m$ is large enough, the coefficient of $g^{2 m}$ is determined by the singularity near the origin. Then, we obtain the large order behavior of $Z_{\mathrm{P}}\left(g^{2}\right)$ as

$$
\begin{aligned}
& Z_{\mathrm{P}}\left(g^{2}\right)=\sum_{m=1}^{\infty} z_{m} g^{2 m} \\
& z_{m} \stackrel{m \rightarrow \infty}{\sim}-\frac{1}{\pi} \int_{0}^{\infty} d z \frac{\operatorname{Im} Z_{\mathrm{NP}}(z)}{z^{m+1}}
\end{aligned}
$$

which reproduces the formula in [15].

We find that $Z_{\mathrm{NP}}$ also reproduces the old result for the instanton-anti-instanton contribution as follows: For weak coupling, the dominate contribution of the integral of $Z_{\mathrm{NP}}$ comes from $t=1 / 3$. Then if we expand $F(t)$ as

$$
F(t)=-\frac{1}{t-1 / 3}+\cdots
$$

and use $x=1 / 3-2 e^{-T}+\cdots$, we obtain

$$
Z_{\mathrm{NP}}=\text { const. } \times \frac{T}{\sqrt{g^{2}}} e^{-1 / 3 g^{2}}\left[T-\ln \left(-2 / g^{2}\right)-\gamma\right]
$$

where $\gamma$ is the Euler's constant. This is the exactly same as the old result 4 . An advantage of our viewpoint is that it clarifies the meaning of a formal analytic continuation in the coupling constant [4, 5]. For this purpose, we perform an

\footnotetext{
${ }^{3}$ This result holds for a sufficiently large $m$ even if $Z_{\mathrm{P}}(z)$ has power divergence for $|z| \rightarrow \infty$ which necessitates subtractions for the dispersion relation Eq.(14). We would like to thank Prof. S. Matsuda for bringing this point to our attention.
} 
analytic continuation for $Z_{\mathrm{NP}}\left(g^{2}\right)$. We denote the contour for $Z_{\mathrm{NP}}\left(\left|g^{2}\right| e^{i \theta}\right)$ as $C_{\mathrm{NP}}(\theta)$. If $\theta$ is changed from zero to $\pi$, the contour $C_{\mathrm{NP}}(0)$ changes to $C_{\mathrm{NP}}(\pi)$ (see Fig.尹) and in the weak coupling limit, $C_{\mathrm{NP}}(\pi)$ can be safely replaced with $C_{\mathrm{V}}$. The resultant integral coincides with that obtained by the formal analytic continuation for $Z\left(g^{2}\right) ; Z_{\mathrm{NP}}\left(\left|g^{2}\right| e^{i \pi}\right)=Z\left(\left|g^{2}\right| e^{i \pi}\right)$. Therefore, in our viewpoint, the formal analytic continuation for $Z\left(g^{2}\right)$ is naturally justified as the real analytic continuation for $Z_{\mathrm{NP}}\left(g^{2}\right)$.

For $\epsilon \neq 0$ case, although some complications arise, the main result does not change. Especially, Eq.(16) and (17) also hold in this case.

In general, there exist valleys which connect the classical minima to the multivalley-instantons. These play important roles in the calculation of the spectrum of the excited states. In the following, we incorporate them into the partition function, $\lim _{T \rightarrow \infty} \operatorname{Tr}\left(e^{-H T}\right)$, which we also denote by $Z$. We have not yet completed the analogous analysis of the I-A valley, but the present approach is sufficient to evaluate the non-perturbative part $Z_{\mathrm{NP}}$ in the weak coupling limit.

When all the valley-instantons are well separated from each other, the action of the multi-valley-instantons is derived by the standard technique [4, 16]. If the valley consists of $n$ pairs of the (anti-)valley-instantons, we obtain

$$
S=\frac{n}{3 g^{2}}-\epsilon \sum_{i=1}^{n} R_{i}-\frac{2}{g^{2}} \sum_{i=1}^{n} e^{-R_{i}}-\frac{2}{g^{2}} \sum_{i=1}^{n} e^{-\tilde{R}_{i}},
$$

where $R_{i}$ is the distance between the $i$-th valley-instanton and the $i$-th anti-valleyinstanton and $\tilde{R}_{i}$ is that between the $i$-th anti-valley-instanton and the $(i+1)$ th valley-instanton. When $g^{2}=\left|g^{2}\right| e^{i \pi}$ and $|g|^{2} \ll 1$, the following equation is meaningful since the interaction between the valley-instanton and the antivalley-instanton is repulsive in this case. (Note that the interaction $\epsilon \sum R_{i}$ is not attractive since $\epsilon \sum R_{i}=\epsilon T-\epsilon \sum \tilde{R}_{i}$. See Eq.(22) below.)

$$
Z=\lim _{T \rightarrow \infty} \sum_{n=1}^{\infty} \alpha^{2 n} J_{n}(T)
$$

where $\alpha=e^{-1 / 6 g^{2}} / g \pi^{1 / 2}$, and

$$
\begin{aligned}
J_{n}(T)= & \frac{T}{n} \int_{0}^{\infty} \prod_{i=1}^{n} d R_{i} d \tilde{R}_{i} \delta\left(\sum_{i=1}^{n}\left(R_{i}+\tilde{R}_{i}\right)-T\right) \\
& \times \exp \left(\epsilon \sum_{i=1}^{n} R_{i}+\frac{2}{g^{2}} \sum_{i=1}^{n} e^{-R_{i}}+\frac{2}{g^{2}} \sum_{i=1}^{n} e^{-\tilde{R}_{i}}\right) .
\end{aligned}
$$

One lesson to draw from the analysis of the I-A valley is that $Z$ is equal to the non-perturbative amplitude $Z_{\mathrm{NP}}$ when $g^{2}=\left|g^{2}\right| e^{i \pi}$ and $\left|g^{2}\right| \ll 1$. Turning back to $\theta=0$ after the evaluation of $Z$, we obtain $Z_{\mathrm{NP}}$ for positive $g^{2}$. 
We extend the Zinn-Justin method [5] to evaluate Eq.(21). If we rewrite the delta function as

$$
\delta\left(\sum_{i=1}^{n}\left(R_{i}+\tilde{R}_{i}\right)-T\right)=\frac{1}{2 \pi i} \int_{-i \infty-\eta}^{i \infty-\eta} d s \exp \left(-s T+s \sum_{i=1}^{n}\left(R_{i}+\tilde{R}_{i}\right)\right),
$$

the integrals over $R_{i}$ and $\tilde{R}_{i}$ are factorized. After summation over multi-valleyinstanton contributions and turning back to $\theta=0$, we finally obtain

$$
Z_{\mathrm{NP}}=-\lim _{T \rightarrow \infty} \frac{1}{2 \pi i} \int_{-i \infty-\eta}^{i \infty-\eta} d s e^{-T s} \frac{\phi^{\prime}(s)}{\phi(s)},
$$

where

$$
\begin{aligned}
\phi(s) & =1-\alpha^{2} \int_{0}^{\infty} d R d \tilde{R} \exp \left((s+\epsilon) R+\frac{2}{g^{2}} e^{-R}+s \tilde{R}+\frac{2}{g^{2}} e^{-\tilde{R}}\right) \\
& =1-\alpha^{2}\left(-\frac{2}{g^{2}}\right)^{2 s+\epsilon} \Gamma(-s-\epsilon) \Gamma(-s) .
\end{aligned}
$$

If we denote the poles corresponding to the solutions of the equation $\phi(s)=0$ as $s_{n}$, we obtain

$$
Z_{\mathrm{NP}} \sim \sum_{n} e^{-s_{n} T}
$$

Therefore, $s_{n}$ give the non-perturbative contribution to the energy levels.

For small coupling, $s_{n}$ can be obtained as a perturbative series in $\alpha$. For $\epsilon$ away from integer values, it yields the following to the first nontrivial order, $\alpha^{2}$;

$$
\begin{aligned}
& E_{\mathrm{NP}}^{(+)}(\epsilon, N)=\frac{1}{2}+N+a^{(+)}(\epsilon, N) \alpha^{2}, \\
& E_{\mathrm{NP}}^{(-)}(\epsilon, N)=-\epsilon+\frac{1}{2}+N+a^{(-)}(\epsilon, N) \alpha^{2},
\end{aligned}
$$

where

$$
a^{( \pm)}(\epsilon, N)=\frac{(-1)^{N+1}}{N !} \Gamma(\mp \epsilon-N)\left(-\frac{2}{g^{2}}\right)^{ \pm \epsilon+2 N} .
$$

The energies (27) are for the localized states in the left well and the right well, respectively, as can be seen in the free limit, $\alpha \rightarrow 0$. For $\epsilon=N_{0}(=0,1,2, \cdots)$, only $E_{\mathrm{NP}}^{(-)}(\epsilon, N)$ for $N \leq N_{0}$ in the above is valid. The rest has to be solved taking into account the confluence of the poles of the two $\Gamma$-functions in Eq.(25), which yields the following to the order $\alpha^{2}$;

$$
\begin{aligned}
& E_{\mathrm{NP}}\left(N_{0}, N, \pm\right)=\frac{1}{2}+N \pm \alpha \sqrt{\frac{1}{N !\left(N+N_{0}\right) !}\left(\frac{2}{g^{2}}\right)^{2 N+N_{0}}} \\
& \quad+\frac{\alpha^{2}}{2}\left(\frac{2}{g^{2}}\right)^{2 N+N_{0}} \frac{1}{N !\left(N+N_{0}\right) !}\left(2 \ln \left(-\frac{2}{g^{2}}\right)+2 \gamma-\sum_{n=1}^{N} \frac{1}{n}-\sum_{n=1}^{N+N_{0}} \frac{1}{n}\right) .
\end{aligned}
$$


The plus and the minus signs in the above expression Eq.(29) correspond to two linear combinations of the perturbative states in the left and right well with the same zero-th order energy. This situation is analogous to the lifting of the degeneracy by the instanton contribution for the symmetric double-well potential.

The expression of the nonperturbative contribution to the energy levels, Eq.(27) - Eq.(29), contain imaginary parts, which are of order $\alpha^{2}$ and are continuous at integer $\epsilon$ 's. Using Eq.(17), these imaginary parts lead to the following leading term of the $m$-th order perturbative coefficient of the $N$-th level, $E_{\mathrm{P}}^{( \pm)}(\epsilon, N, m)$ $\left(E_{\mathrm{P}}^{( \pm)}(\epsilon, N)=\sum_{m=0}^{\infty} E_{\mathrm{P}}^{( \pm)}(\epsilon, N, m) g^{2 m}\right)$;

$$
\begin{aligned}
E_{\mathrm{P}}^{( \pm)}(\epsilon, N, m) & =A^{( \pm)}(\epsilon, N) 3^{m} \Gamma( \pm \epsilon+2 N+m+1)\left[1+O\left(\frac{1}{m}\right)\right] \\
A^{( \pm)}(\epsilon, N) & \equiv-\frac{3}{\pi} \frac{6^{ \pm \epsilon+2 N}}{N ! \Gamma( \pm \epsilon+1+N)} .
\end{aligned}
$$

The expression for $E_{\mathrm{P}}^{(-)}(\epsilon, 1)$ coincides with the expression obtained in Ref.[11, [17].

We have independently carried out the numerical and exact calculation of the perturbative coefficients $E_{\mathrm{P}}^{( \pm)}(\epsilon, N, m)$ by the methods described in Ref. 11, 15, 18] to the 200-th order for the following four categories; (a) $N=0(-)$ level (ground state) for $\epsilon=0$ to 10 with $\Delta \epsilon=0.2$ interval, (b) $N=0$ (+) level for $\epsilon=0$ to 20 with $\Delta \epsilon=0.2$, (c) $N=3$ (+)-level up to $\epsilon=6.5$ with $\Delta \epsilon=0.5$, (d) $N=1$ to $6(-)$ levels for $\epsilon=2.5$. In order to check the leading $m$-dependent terms in Eq.(30), we take their ratio,

$$
\frac{E_{\mathrm{P}}^{( \pm)}(\epsilon, N, m)}{E_{\mathrm{P}}^{( \pm)}(\epsilon, N, m-1)}=3( \pm \epsilon+m) .
$$

The agreement between the numerical fitting of the perturbative coefficients for $m=150$ to 200 and this theoretical prediction is excellent in all the calculated cases, with the maximum error of order $0.1 \%$. Next we have calculated $A^{( \pm)}(\epsilon, N)$ defined by Eq.(30) numerically and compared with Eq.(31). The result for the case (a) is plotted in Fig. 35. The difference between Eq.(31) and the calculated value is at most $0.1 \%$ (at $\epsilon=9.8$ ). For the case (b), the error is at most $15 \%$ (at $\epsilon=20$ ). For the cases (c) and (d), the maximum error is $0.15 \%$. In summary, the agreement between the expression (30), (31), and the actual perturbative coefficients is excellent in all the cases examined.

The reader may note that $A^{(-)}(\epsilon, 0)$ is zero for any positive integer $\epsilon$. For $\epsilon=1$, this is because of the supersymmetry, which prohibits any perturbative correction to the ground state energy [9]. For $\epsilon=2$, there are no perturbative corrections to the energy levels of the ground state and the first excited state due to the supersymmetry [11]. We have calculated these energy levels numerically and compared them with our valley result, Eq.(27) - Eq.(29). The result is plotted 
in Fig. 4 and the agreement is excellent. For $\epsilon=3,4, \cdots$, there are analogues of supersymmetry that explain vanishing of $A^{(-)}$and also some other results for excited states. These results will be published in near future.

We stress that the bounce solution plays no important roles in the large order behavior of the perturbative series. As a result of this, states that have no associated quantum tunneling phenomena do not necessarily have Borel-summable perturbative series, in contrast to some beliefs [6]. In fact, non Borel-summability is found for low-lying stable states when $\epsilon \neq$ integer, which is confirmed by the calculation of the perturbative coefficients.

We believe that our analysis clearly shows that the valley is essential for the definition of the so-called "non-perturbative effects"; only when it is defined in the light of the separation from the valley, it becomes physically sensible and calculable. Same can be said of the perturbation theory; the origin of its Borel singularity and its cancellation by the non-perturbative effects become evident in view of the valley.

H. Aoyama's work is supported in part by the Grant-in-Aid for Scientific Research (C)-07640391 and 09226219. H. Kikuchi's work is supported in part by the Grant-in-Aid for Scientific Research 09226232. M. Sato and S. Wada's work is supported in part by the Grant-in-Aid for JSPS fellows. Numerical computation in this work was in part supported by the Yukawa Institute for Theoretical Physics.

\section{References}

[1] F. J. Dyson, Phys. Rev. 85 (1952) 631.

[2] For the review, see "Large-Order Behaviour of Perturbation Theory", ed. by J. C. Le Guillou and J. Zinn-Justin, (North-Holland, 1990), and references cited therein.

[3] E. Brézin, G. Parisi, and J. Zinn-Justin, Phys. Rev. D16 (1977) 170.

[4] E. B. Bogomolny, Phys. Lett. B91 (1980) 431.

[5] J. Zinn-Justin, Nucl. Phys. B192 (1981) 125; Nucl. Phys. B218 (1983) 333.

[6] E. Brézin, J. C. Le Guillou, and J. Zinn-Justin, Phys. Rev. D15 (1977) 1558.

[7] H. Aoyama, H. Kikuchi, T. Harano, M. Sato, S. Wada, hep-th/9606159 (To appear in Phys. Rev. Lett.)

[8] H. Aoyama, H. Kikuchi, T. Harano, I. Okouchi, M. Sato, S. Wada, Prog. Theor. Phys. Supplement 127 (1997) 1. 
[9] P. Salomonson and J. W. van Holten, Nucl. Phys. B196 (1982) 509.

[10] I. I. Balitsky and A.V. Yung, Nucl. Phys. B274 (1986) 475.

[11] J. I. Verbaarschot, P. West, and Tai Tsun Wu, Phys. Rev. D42 (1990) 1276.

[12] I. I. Balitsky and A.V. Yung, Phys. Lett. B168 (1986) 13.

[13] H. Aoyama and H. Kikuchi, Nucl. Phys. B369 (1992) 219.

[14] H. Kikuchi, Phys. Rev. D45 (1991) 1240.

[15] J. Zinn-Justin, J. Math. Phys. 25 (1984) 549.

[16] H. Aoyama and H. Kikuchi, Phys. Lett. B247 (1990) 75, Phys. Rev. D43 (1991) 1999, Int. Mod. Phys. A7 (1992) 2741.

[17] J. I. Verbaarschot and P. West, Phys. Rev. D43 (1991) 2718.

[18] E. Brézin, G. Parisi, and J. Zinn-Justin, Phys. Rev. D16 (1977) 408. 


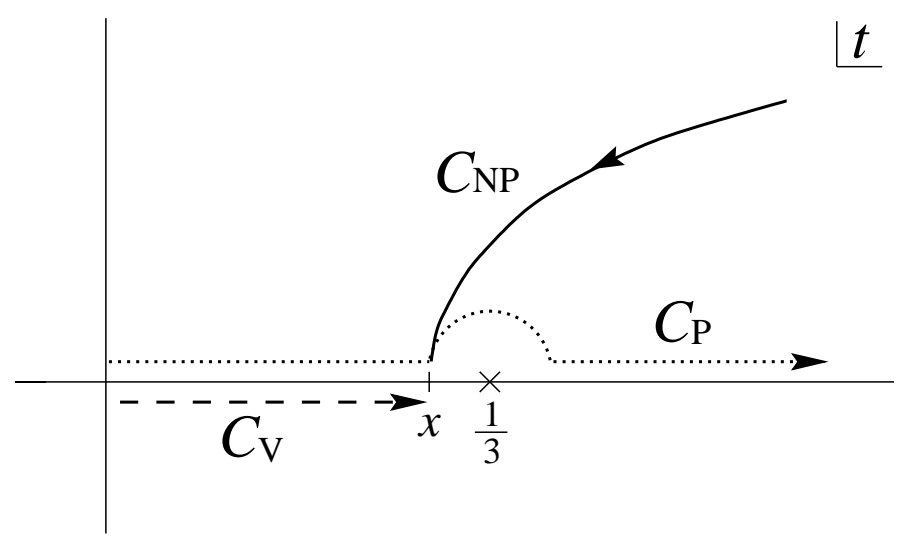

Figure 1: Deformation of the contour $C_{V}$ to the sum of $C_{\mathrm{P}}$ and $C_{\mathrm{NP}}$.

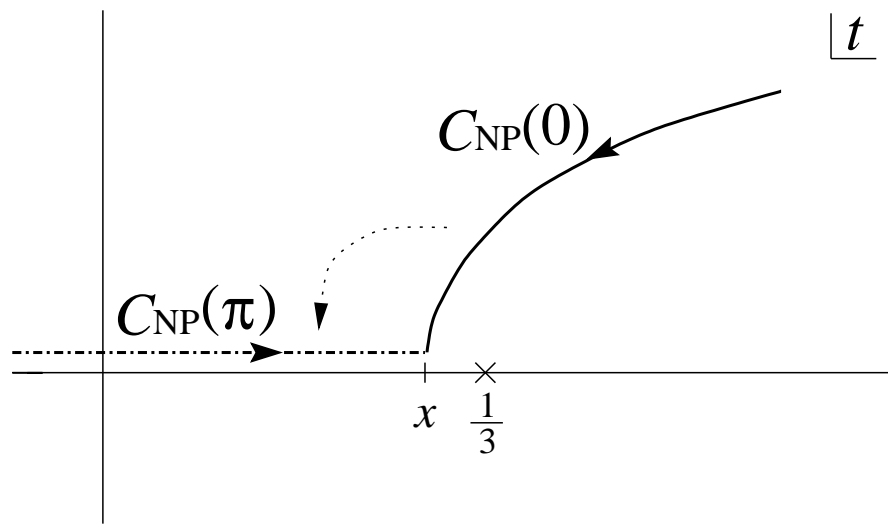

Figure 2: The change of the contour $C_{\mathrm{NP}}(\theta)$ as $\theta$ is changed from zero to $\pi$. 


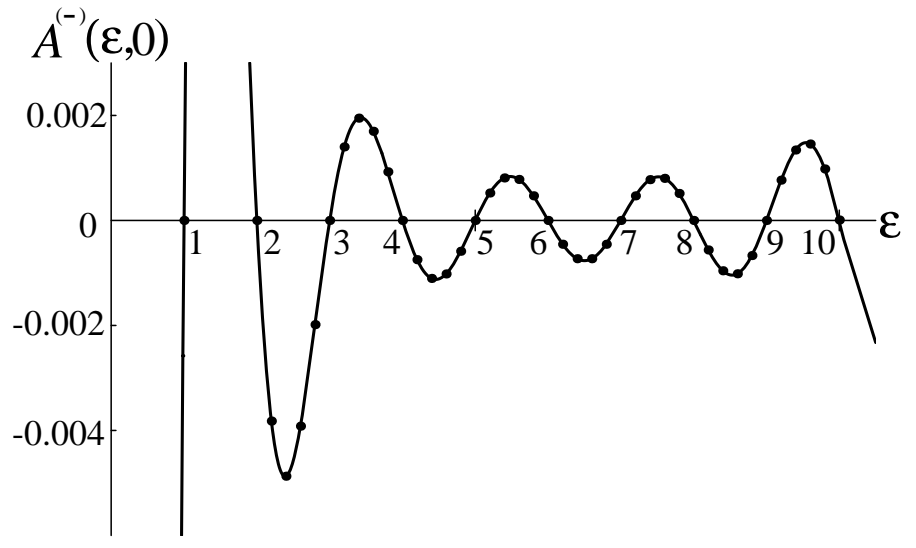

Figure 3: Comparison of the fitting to the perturbative coefficients (indicated by dots) and the theoretical prediction (solid line) of $A^{(-)}(\epsilon, 0)$.

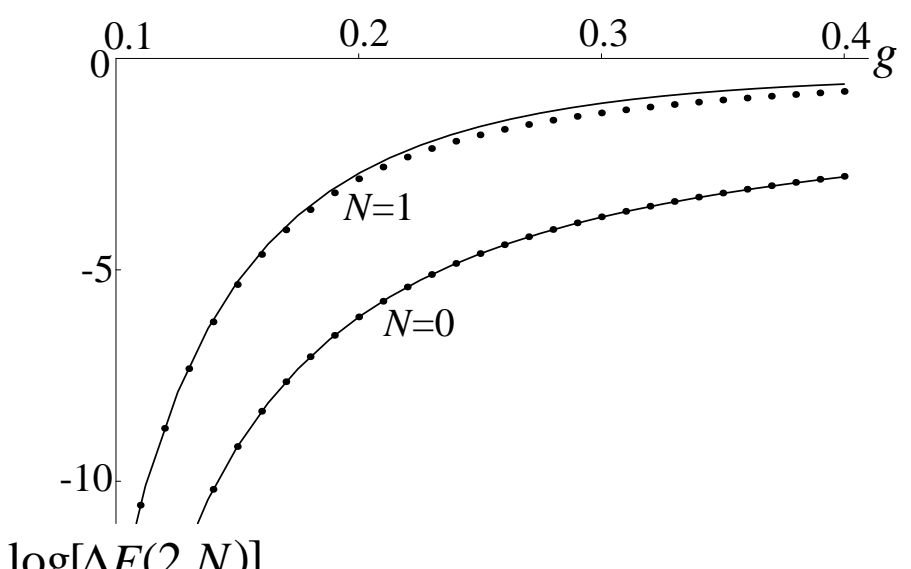

Figure 4: Comparison of our valley results and the numerical results for the energy levels of the ground state and the first excited states at $\epsilon=2 . \Delta E$ is the difference between the energy level and the zero-th order results. The solid line is our result, while the dots represent the numerical results. 